\title{
STABILITY ANALYSIS OF SPECTRAL METHODS FOR HYPERBOLIC INITIAL-BOUNDARY VALUE SYSTEMS*
}

\author{
DAVID GOTTLIEB $\ddagger \S$, LIVIU LUSTMAN $\ddagger$ AND EITAN TADMOR $\ddagger \ddagger \rrbracket$
}

\begin{abstract}
We treat a constant coefficient hyperbolic system in one space variable, with zero initial data. Dissipative boundary conditions are imposed at the two points $x= \pm 1$. This problem is discretized either by a spectral or pseudospectral approximation in space. We demonstrate sufficient conditions under which the spectral numerical solution is stable; moreover, these conditions have to be checked only for scalar equations. The stability theorems take the form of explicit bounds for the norm of the solution in terms of the boundary data. The dependence of these bounds on $N$, the number of points in the domain (or equivalently the degree of the polynomials involved), is investigated for a class of standard spectral methods, including Chebyshev and Legendre collocations.
\end{abstract}

Key words. hyperbolic systems, boundary conditions, spectral methods, stability

AMS(MOS) subject classifications. 65M10, 65N35

1. Introduction. In this paper we study the stability of spectral polynomial methods for the approximation of initial-boundary value hyperbolic systems with constant coefficients and dissipative boundary conditions (Assumption I). We show that any particular spectral method is stable when applied to a system, if it satisfies certain conditions (Assumptions II and III) for the corresponding scalar problem.

Our treatment follows closely the approach of Kreiss [4], leading to an algebraic condition. However, in the spectral method the requirement that the approximate solution be a polynomial produces a different algebraic problem from the standard finite difference discretizations.

We should also review briefly the stability results for spectral methods and hyperbolic systems. The stability of Chebyshev approximation of the scalar problem

$$
u_{t}=u_{x}, \quad u(x=1, t)=0
$$

was proved in [2] for the Galerkin and tau-methods, and in [7] for the collocation method. The same proofs hold for the Legendre method; they are based on energy estimates, showing that the norm

$$
\int_{-1}^{1}(1+x) w(x) u^{2}(x, t) d x
$$

is bounded by the data. Here the weight $w(x)$ depends on the discretization used and equals $\left(1-x^{2}\right)^{-1 / 2}$ for Chebyshev, $w(x)=1$ for Legendre.

* Received by the editors January 21, 1986; accepted for publication (in revised form) March 31, 1986. This research was supported in part by the National Aeronautics and Space Administration under NASA contracts NASl-17070 and NASl-18107 while the authors were in residence at ICASE, NASA Langley Research Center, Hampton, Virginia 23665-5225. Additional support was also provided in part by Air Force Office of Scientific Research grant AFOSR-83-0089 for the first author, and by Natural Science Foundation grant DMS85-03294 and Army Research Office grant DAAG29-85-K-0190 for the third author while in residence at University of California, Los Angeles, California 90024.

$\dagger$ School of Mathematical Sciences, Tel-Aviv University, Tel-Aviv, Israel.

$\ddagger$ Institute for Computer Applications in Science and Engineering, NASA Langley Research Center, Hampton, Virginia 23665-5225.

§ Division of Applied Mathematics, Brown University, Providence, Rhode Island 02912.

I This author is a Bat-Sheva Foundation Fellow. 
Reyna [8] suggested a stable numerical method for strictly hyperbolic systems with maximal dissipative boundary conditions, based on high modes smoothing. His result applies to problems with variable coefficients. While his procedure ensures $l_{2}$ stability, our constant coefficients proof requires no smoothing; instead stability is obtained here in certain weighted $l_{2}$ norms.

The first results about hyperbolic systems with general dissipative boundary conditions were reported in [5], where it is shown that the numerical solutions decay in time, thus mimicking the differential solutions.

In the present paper we combine the above results into a general stability theory for the stability of spectral methods. A future paper will discuss the convergence of the spectral approximations to the exact solution.

Definitions. Consider the first order hyperbolic system of partial differential equations

$$
\frac{\partial \mathrm{u}}{\partial t}=A \frac{\partial \mathrm{u}}{\partial x}, \quad-1 \leqq x \leqq 1, \quad t \geqq 0 .
$$

Here,

$$
\mathbf{u}=\mathbf{u}(x, t)=\left(u^{(1)}(x, t), \cdots, u^{(n)}(x, t)\right)^{\prime}
$$

is the vector of unknowns and $A$ is a fixed $n \times n$ coefficient matrix. Since by hyperbolicity $A$ is similar to a real diagonal matrix, we may assume without loss of generality that it is diagonal:

$$
A=\left(\begin{array}{cc}
A^{I} & 0 \\
0 & A^{I I}
\end{array}\right), \quad A^{I}=\left(\begin{array}{ccc}
a_{1} & & \\
& \ddots & \\
& & a_{l}
\end{array}\right)<0, \quad A^{I I}=\left(\begin{array}{ccc}
a_{l+1} & & \\
& \ddots & \\
& & a_{n}
\end{array}\right)>0 .
$$

The solution of this system is uniquely determined if we prescribe initial conditions

$$
\mathbf{u}(x, 0)=0, \quad-1 \leqq x \leqq 1
$$

and boundary conditions

$$
\begin{aligned}
& \mathbf{u}^{I}(-1, t)=L \mathbf{u}^{I I}(-1, t)+\mathrm{g}^{I}(t), \\
& \mathbf{u}^{I I}(1, t)=R \mathbf{u}^{I}(1, t)+\mathrm{g}^{I I}(t), \quad t \geqq 0 .
\end{aligned}
$$

In these formulas,

$$
\mathbf{g}=\mathbf{g}(t)=\left(\mathbf{g}^{I}(t), \mathbf{g}^{I I}(t)\right)^{\prime}
$$

is a given $n$-vector, and

$$
\mathbf{u}^{I}=\left(u^{(1)}, \cdots, u^{(l)}\right)^{\prime}, \quad \mathbf{u}^{I I}=\left(u^{(l+1)}, \cdots, u^{(n)}\right)^{\prime}
$$

is a partition of $\mathbf{u}$ into its inflow and outflow components-corresponding to the partition of $A$ in (1.1b) -while $L$ and $R$ are constant reflection matrices of order $l \times(n-l)$ and $(n-l) \times l$, respectively.

The system (1.1a)-(1.1e) is a well-posed problem in the sense that it satisfies an a priori energy estimate which we now describe. Define the spatial norm associated with a pair of positive weight functions $\omega(x)=\left(\omega^{I}(x), \omega^{I I}(x)\right)$,

$$
\|\mathrm{u}(x)\|^{2} \equiv\|\mathbf{u}(x)\|_{\omega}^{2}=\int_{-1}^{1}\left|\mathbf{u}^{I}(x)\right|^{2} \omega^{I}(x) d x+\int_{-1}^{1}\left|\mathbf{u}^{I I}(x)\right|^{2} \omega^{I I}(x) d x .
$$

Here and elsewhere in the paper we denote by $|\mathbf{v}|$ the Euclidean norms of a vector $\mathbf{v}$; similarly $|\boldsymbol{A}|=\sup |\boldsymbol{A v}| /|\mathbf{v}|$. 
Then, for well-posedness one must obtain the following inequality, with some $\eta_{0} \geqq 0$ :

$$
\left(\eta-\eta_{0}\right) \int_{t=0}^{\infty} e^{-2\left(\eta-\eta_{0}\right)^{t}}\|\mathrm{u}(x, t)\|^{2} d t \leqq \text { const } \int_{t=0}^{\infty} e^{-2\left(\eta-\eta_{0}\right)^{t}}|\mathbf{g}(t)|^{2} d t
$$

for all $\eta>\eta_{0}$.

The parameter $\eta_{0}$ measures the exponential time growth of the solution. We are interested in the case where there is no growth in time, and therefore postulate the following dissipativity requirement:

Assumption I. There exists a constant $\delta>0$ such that

$$
|R| \cdot|L| \leqq 1-\delta<1 \text {. }
$$

The inequality (1.3) guarantees that waves originating at one of the boundaries are not amplified when reflected at the other one. Consequently, there is no time growth; in Appendix A we show that there is a weight $\omega$ such that for any $\eta>0$

$$
\eta \int_{t=0}^{\infty} e^{-2 \eta t}\|u(x, t)\|^{2} d t \leqq \text { const } \int_{t=0}^{\infty} e^{-2 \eta t}|\mathbf{g}(t)|^{2} d t,
$$

i.e., $\eta_{0}$ in formula (1.2b) may be taken as zero in this dissipative case.

We study polynomial spectral and pseudospectral discretizations of (1.1). In any such approximation, one seeks a vector of $\mathrm{N}$-degree polynomials

$$
\mathbf{v} \equiv \mathbf{v}_{N}(x, t)=\left(v_{N}^{(1)}(x, t), \cdots, v_{N}^{(n)}(x, t)\right)^{\prime}
$$

such that

$$
\frac{\partial \mathbf{v}}{\partial t}=A \frac{\partial \mathbf{v}}{\partial x}+Q(x) \tau
$$

Here, $Q(x)$ is a diagonal matrix of the form

$$
Q(x)=\left(\begin{array}{cc}
q^{I}(x) I_{l \times l} & 0 \\
0 & q^{I I}(x) I_{(n-l) \times(n-l)}
\end{array}\right)
$$

where $q^{I}(x), q^{I I}(x)$ are the $N$-degree polynomials that characterize the specific (pseudo)spectral method employed, and

$$
\tau \equiv \tau(t)=\left(\tau^{I}, \tau^{I I}\right)^{\prime}
$$

is an $n$-vector to be determined by the set of boundary conditions $(1.1 \mathrm{~d})$ :

$$
\mathbf{v}^{I}(-1, t)=L \mathbf{v}^{I I}(-1, t)+\mathbf{g}^{I}(t), \quad \mathbf{v}^{I I}(1, t)=R \mathbf{v}^{I}(1, t)+\mathrm{g}^{I I}(t) .
$$

We shall call $q^{I}(x), q^{I I}(x)$ the forcing polynomials, since they appear as inhomogeneous terms in the discretization of the originally homogeneous system (1.1). Some examples are in order.

In the spectral Galerkin-Chebyshev method, one has

$$
\begin{aligned}
& q^{I}(x)=\frac{1}{2} T_{0}(x)+\sum_{k=1}^{N}(-1)^{k} T_{k}(x), \\
& q^{I I}(x)=\frac{1}{2} T_{0}(x)+\sum_{k=1}^{N} T_{k}(x)
\end{aligned}
$$

with $T_{k}(x)$ denoting the $k$ th Chebyshev polynomial. 
In the pseudospectral Chebyshev method, one may collocate only at the interior extrema of $T_{N+1}(x)$, yielding

$$
q^{I}(x)=q^{I I}(x)=T_{N+1}^{\prime}(x)
$$

or use as collection points these extrema together with the downstream boundaries, in which case

$$
q^{I}(x)=(1-x) T_{N}^{\prime}(x), \quad q^{I I}(x)=(1+x) T_{N}^{\prime}(x) .
$$

Similarly, in the pseudospectral Legendre case one has

$$
q^{I}(x)=q^{I I}(x)=P_{N+1}^{\prime}(x)
$$

or alternatively

$$
q^{I}(x)=(1-x) P_{N}^{\prime}(x), \quad q^{I I}(x)=(1+x) P_{N}^{\prime}(x)
$$

with $P_{k}(x)$ denoting the $k$ th Legendre polynomial. These and other examples are outlined in [2], [3].

In this paper we provide a stability study for the (pseudo)spectral method (1.5). We define stability in terms of an a priori energy estimate analogous to the differential one:

Definition 1.1 (Stability). The approximation (1.5a)-(1.5c) is stable if there exist a weighting pair $\omega(x)$ and constants $\alpha$ and $\eta_{0} \geqq 0$ such that for all $\eta>\eta_{0}$ we have

$$
\left(\eta-\eta_{0}\right) \int_{t=0}^{\infty} e^{-2\left(\eta-\eta_{0}\right) t}\left\|\mathbf{v}_{N}(x, t)\right\|^{2} d t \leqq \text { const } N^{2 \alpha} \int_{t=0}^{\infty} e^{-2\left(\eta-\eta_{0}\right) t}|\mathbf{g}(t)|^{2} d t .
$$

We note that the exponential time growth factor here, $\eta_{0}$, need not be the same as the one in the differential estimate (1.2b). The other constant, $\alpha$, accounts for a possible algebraically increasing dependence on the discretization parameter $N$.

To present our results, let us introduce into (1.5) a new variable $e^{-\eta t} \mathbf{v}_{N}(x, t)$ and, following the procedure of [4], Fourier-transform the resulting equations with respect to time. Denote by $\xi$ the real dual variable corresponding to $t$, and by

$$
\begin{aligned}
& \hat{\mathbf{v}} \equiv \hat{\mathbf{v}}_{N}(x, s)=\mathscr{F}\left(e^{-\eta t} \mathbf{v}_{N}(x, t)\right), \\
& \hat{\mathbf{g}} \equiv \hat{\mathbf{g}}(s)=\mathscr{F}\left(e^{-\eta t} \mathbf{g}(t)\right), \quad s=\eta+i \xi
\end{aligned}
$$

the transforms of $e^{-\eta t} \mathbf{v}_{N}$ and $e^{-\eta t} \mathbf{g}$, respectively (these functions are set to zero for $t<0)$. The resulting equation for $\hat{\mathbf{v}}=\left(\hat{\mathbf{v}}^{I}(x, s), \hat{\mathbf{v}}^{I I}(x, s)\right)^{\prime}$ is then

$$
s \hat{\mathbf{v}}^{I}=A^{I} \frac{d \hat{\mathrm{v}}^{I}}{d x}+q^{I}(x) \tau^{I}, \quad s \hat{\mathbf{v}}^{I I}=A^{I I} \frac{d \hat{\mathrm{v}}^{I I}}{d x}+q^{I I}(x) \tau^{I I}
$$

where the $n$-vector $\tau \equiv \tau(s)=\left(\tau^{I}, \tau^{I I}\right)^{\prime}$ is to be determined from the boundary conditions

$$
\hat{\mathbf{v}}^{I}(-1, s)=L \hat{\mathbf{v}}^{I I}(-1, s)+\hat{\mathbf{g}}^{I}(s), \quad \hat{\mathbf{v}}^{I I}(1, s)=R \hat{\mathbf{v}}^{I}(1, s)+\hat{\mathbf{g}}^{I I}(s) .
$$

Using Parseval's relation, we conclude:

LEMMA 1.2. The approximation is stable if there exist a weighting pair $\omega(x)$ and constants $\alpha$ and $\eta_{0} \geqq 0$ such that for all $s$ with $\operatorname{Re} s=\eta>\eta_{0}$ the following holds

$$
\left(\eta-\eta_{0}\right)\left\|\hat{\mathbf{v}}_{N}(x, s)\right\|^{2} \leqq \text { const } N^{2 \alpha}|\hat{\mathbf{g}}(s)|^{2} .
$$

In $\S \S 2$ and 3 we discuss necessary conditions for the stability estimate (1.14). One such condition is the obvious requirement that the (pseudo)spectral method must be stable for a scalar problem. Another one is that the homogeneous two-point boundary 
value problem (1.13) should have no eigenvalues with $\operatorname{Re} s>\eta_{0}$. In $\S 4$ we show that a strengthened version of these necessary conditions guarantees stability. The novelty of our sufficient stability criterion is that it deals exclusively with the properties of the scalar model and there is no need to consider the complicated coupling of such scalar equations through the boundary conditions (1.5c).

In the last section we demonstrate various important cases in which our sufficient conditions for stability are satisfied and, thus, deduce stability theorems for pseudospectral discretizations of the initial-boundary value system (1.1).

2. The scalar problem. The system (1.13a) decouples into $n$ scalar equations of the form

$$
s \hat{v}=a \frac{d \hat{v}}{d x}+q(x) \tau, \quad-1 \leqq x \leqq 1
$$

Here $\hat{v} \equiv \hat{v}_{N}(x, s)$ and $a$, stand for any of the corresponding components of $\hat{\mathbf{v}}_{N}(x, s)$ and the diagonal of $A, q(x)$ equals either $q^{I}(x)$ for $a<0$, or $q^{I I}(x)$ for $a>0$, and $\tau=\tau(s)$ is to be determined by boundary conditions. In the scalar case, these boundary conditions amount to prescribing the upstream values

$$
\begin{array}{ll}
\hat{v}_{N}(-1, s)=g(s), & a<0, \\
\hat{v}_{N}(1, s)=g(s), & a>0 .
\end{array}
$$

If formula (1.14) is valid, a similar stability estimate holds for (2.1). We make the necessary

Assumption II (Scalar stability). There exist a weighting pair $\omega^{I}(x), \omega^{I I}(x)$ and constants $\alpha$ and $\eta_{0} \geqq 0$, such that for all $s$ with $\operatorname{Re} s=\eta>\eta_{0}$, we have

$$
\begin{aligned}
& \left(\eta-\eta_{0}\right)\left\|\hat{v}_{N}(x, s)\right\|_{\omega^{I}}^{2} \leqq \text { const } N^{2 \alpha}\left|\hat{v}_{N}(-1, s)\right|^{2}, \quad a<0, \\
& \left(\eta-\eta_{0}\right)\left\|\hat{v}_{N}(x, s)\right\|_{\omega^{I I}}^{2} \leqq \text { const } N^{2 \alpha}\left|\hat{v}_{N}(1, s)\right|^{2}, \quad a>0 .
\end{aligned}
$$

3. The eigenvalue problem. Connected with (1.13) is the following eigenvalue problem:

Let $\mathbb{P}_{N}$ be the space of all polynomials of degree $\leqq N$. We say that $\phi \in \mathbb{P}_{N}$ is an eigenfunction of (1.13) corresponding to an eigenvalue $s$, if $\phi \equiv \phi_{N}(x, s)=\left(\phi^{I}, \phi^{I I}\right)^{\prime}$ is a nontrivial solution of

$$
\mathrm{s} \phi^{I}=A^{I} \frac{d \phi^{I}}{d x}+q^{I}(x) \tau^{I}, \quad s \phi^{I I}=A^{I I} \frac{d \phi^{I I}}{d x}+q^{I I}(x) \tau^{I I}
$$

with boundary conditions

$$
\phi^{I}(-1, s)=L \phi^{I I}(-1, s), \quad \phi^{I I}(1, s)=R \phi^{I}(1, s) .
$$

In order to determine whether $s \neq 0$ is an eigenvalue, we proceed as follows. Equations (3.1a) form a system of ordinary differential equations in $x$, depending on a parameter $s$,

$$
\left(s I_{n \times n}-A \frac{d}{d x}\right) \phi=Q(x) \tau .
$$

This can be solved by a formal series

$$
\phi(x)=\left(s I-A \frac{d}{d x}\right)^{-1} Q(x) \tau \equiv \sum_{k=0}^{\infty} s^{-k-1} A^{k} Q^{(k)}(x) \tau .
$$


We note that since $Q(x)$ consists of $N$-degree polynomials, the sum on the right include only the first $N+1$ terms. Rewriting this in the usual partitioned form, we have

LemMA 3.1. Let $P^{I}(x, s) \equiv p^{I}\left(x, s ; A^{I}\right)$ and $P^{I I}(x, s) \equiv p^{I I}\left(x, s ; A^{I I}\right)$ denote the diagonal matrices

$$
\begin{aligned}
& P^{I}(x, s)=\sum_{k=0}^{N} s^{-k-1}\left(q^{I}(x)\right)^{(k)}\left(A^{I}\right)^{k} \\
& P^{I I}(x, s)=\sum_{k=0}^{N} s^{-k-1}\left(q^{I I}(x)\right)^{(k)}\left(A^{I I}\right)^{k} .
\end{aligned}
$$

Then the polynomial solution of $(2.2 \mathrm{a})$ is given by

$$
\phi^{I}(x, s)=P^{I}(x, s) \tau^{I}, \quad \phi^{I I}(x, s)=P^{I I}(x, s) \tau^{I I} .
$$

Substituting this expression into the boundary conditions (3.1b), we end up with the homogeneous linear system:

$$
\left(\begin{array}{cc}
P^{I}(-1, s) & -L P^{I I}(-1, s) \\
-R P^{I}(1, s) & P^{I I}(1, s)
\end{array}\right)\left(\begin{array}{c}
\tau^{I} \\
\tau^{I I}
\end{array}\right)=0 .
$$

Denoting the coefficient matrix by $D(s)$

$$
D(s)=\left(\begin{array}{cc}
P^{I}(-1, s) & -L P^{I I}(-1, s) \\
-R P^{I}(1, s) & P^{I I}(1, s)
\end{array}\right)
$$

we arrive at

LEMMA 3.2. A complex number $s \neq 0$ is an eigenvalue of (3.1), if and only if,

$$
\operatorname{Det}[D(s)]=0 \text {. }
$$

Suppose now that an eigenvalue $s$ exists, with $\operatorname{Re} s=\eta>\eta_{0} \geqq 0$. Then the corresponding eigenfunction is a nonvanishing solution to the homogeneous problem (1.13) with $g=0$, in contradiction to (1.14). For future reference we state this as

LEMMA 3.3. A necessary condition for the stability estimate (1.14) to hold is that the eigenvalue problem (3.1) has no eigenvalues $s$ with $\operatorname{Re} s>\eta_{0} \geqq 0$, i.e.,

$$
\operatorname{Det}[D(s)] \neq 0, \quad \operatorname{Re} s=\eta>\eta_{0} \geqq 0 .
$$

4. The stability estimate. Here we present a stability theorem for hyperbolic systems with initial and boundary value conditions.

In the previous two sections we exhibited two necessary conditions for the stability of (1.5). The first of these, (2.2), is a property of the individual scalar equations which constitute system (1.5a). The second one, (3.6), involves the coupling of these equations through the boundary conditions. Thus, in principle, in order to test this second condition, one should check the boundary determinant of $D(s)$ for each pair of the reflection matrices $L$ and $R$. However, (3.6) is implied by a much simpler condition, which deals with the scalar equations separately and does not depend upon $L$ and $R$. Moreover, this strengthened condition turns out to guarantee stability, as we shall presently prove.

We make the following

Assumption III. The polynomial solution of the scalar inflow problem:

$$
s \hat{v}^{I}=a \frac{d \hat{v}^{I}}{d x}+q^{I}(x), \quad a<0
$$


satisfies

$$
\left|\hat{v}^{I}(1, s)\right| \leqq\left|\hat{v}^{I}(-1, s)\right|, \quad \operatorname{Re} s=\eta>\eta_{0} \geqq 0 .
$$

Similarly, in the outflow scalar case,

$$
s \hat{v}^{I I}=a \frac{d \hat{v}^{I I}}{d x}+q^{I I}(x), \quad a>0,
$$

we have

$$
\left|\hat{v}^{I I}(-1, s)\right| \leqq\left|\hat{v}^{I I}(1, s)\right|, \quad \operatorname{Re} s=\eta>\eta_{0} \geqq 0 .
$$

The estimates (4.1b) and (4.2b) are discrete analogues of differential estimates, as shown in Appendix A, cf. A.11.

We can now prove

LeMMA 4.1. Let Assumptions I, II, and III hold. Then, there exists a constant $\eta_{0} \geqq 0$ such that for all $s$ with $\operatorname{Re} s=\eta>\eta_{0}$ we have

$$
\operatorname{Det}[D(s)] \neq 0 \text {. }
$$

Proof. Consider the diagonal matrix $P^{I}(x, s)$ of $(3.4 a)$. Its $j$ th diagonal element $\hat{p}_{N}^{(j)}(x, s)$ is given by

$$
\hat{p}_{N}^{(j)}(x, s)=\left(s-a_{j} \frac{d}{d x}\right)^{-1} q^{I}(x)
$$

That is, we have

$$
P^{I}(x, s)=\left(\begin{array}{lll}
\hat{p}_{N}^{(1)}(x, s) & & \\
& \ddots & \\
& & \hat{p}_{N}^{(l)}(x, s)
\end{array}\right)
$$

where $\hat{p}^{(j)} \equiv \hat{p}_{N}^{(j)}(x, s)$ satisfies the inflow scalar equation

$$
s \hat{p}^{(j)}=a_{j} \frac{d \hat{p}^{(j)}}{d x}+q^{I}(x), \quad a_{j}<0, \quad 1 \leqq j \leqq l .
$$

Similarly, the diagonal matrix

$$
P^{I I}(x, s)=\left(\begin{array}{lll}
\hat{p}_{N}^{(l+1)}(x, s) & & \\
& \ddots & \\
& & \hat{p}_{N}^{(n)}(x, s)
\end{array}\right)
$$

consists of solutions to the outflow scalar equations

$$
s \hat{p}^{(j)}=a_{j} \frac{d \hat{p}^{(j)}}{d x}+q^{I I}(x), \quad a_{j}>0, \quad l<j \leqq n .
$$

Fix $s$ with $\operatorname{Re} s=\eta>\eta_{0} \geqq 0$. We first claim that both matrices $P^{I}(-1, s)$ and $P^{I I}(1, s)$ are nonsingular. Indeed, if for some index $j, 1 \leqq j \leqq 1, \hat{p}_{N}^{(j)}$ vanishes on the left boundary

$$
\hat{p}_{N}^{(j)}(-1, s)=0 \text {, }
$$

then by the scalar stability Assumption I, see (2.2a), the solution of (4.5a) vanishes everywhere,

$$
\left\|\hat{p}_{N}^{(j)}(x, s)\right\|_{\omega^{I}=0}
$$


This, however, contradicts formula (4.5a). In the same way, one shows that $P^{I I}(1, s)$ is regular. Hence, the matrix $D(s)$ can be factored (here the ratio of two diagonal matrices is understood as the diagonal of quotients):

$$
D(s)=\left(\begin{array}{cc}
P^{I}(-1, s) & -L P^{I I}(-1, s) \\
-R P^{I}(1, s) & P^{I I}(1, s)
\end{array}\right)
$$

$$
=\left(\begin{array}{cc}
I & -L \frac{P^{I I}(-1, s)}{P^{I I}(1, s)} \\
-R \frac{P^{I}(1, s)}{P^{I}(-1, s)} & I
\end{array}\right)\left(\begin{array}{cc}
P^{I}(-1, s) & 0 \\
0 & P^{I I}(1, s)
\end{array}\right) .
$$

Next we prove that the matrix $E$ given by

$$
E(s)=\left(\begin{array}{cc}
I & -L \frac{P^{I I}(-1, s)}{P^{I I}(1, s)} \\
-R \frac{P^{I}(1, s)}{P^{I}(-1, s)} & I
\end{array}\right)
$$

is regular, with a uniformly bounded inverse

$$
\left|E^{-1}(s)\right| \leqq K \equiv \frac{1+|R|+|L|}{\delta}, \quad \operatorname{Re} s=\eta>\eta_{0} \geqq 0 .
$$

It is here that we make use of Assumptions I and III. Denoting

$$
\mathscr{R}=R \frac{P^{I}(1, s)}{P^{I}(-1, s)}, \quad \mathscr{L}=L \frac{P^{I I}(-1, s)}{P^{I I}(1, s)},
$$

we have, by (1.3), (4.1b) and (4.2b)

$$
\left|(I-\mathscr{R} \mathscr{L})^{-1}\right| \leqq \frac{1}{1-|\mathscr{R}||\mathscr{L}|} \leqq \frac{1}{\delta}, \quad\left|(I-\mathscr{L} \mathscr{R})^{-1}\right| \leqq \frac{1}{1-|\mathscr{L}||\mathscr{R}|} \leqq \frac{1}{\delta} .
$$

Hence, $E$ can be inverted:

$$
E^{-1}(s)=\left(\begin{array}{cc}
I & \mathscr{R} \\
\mathscr{L} & I
\end{array}\right)\left(\begin{array}{cc}
\frac{(I-\mathscr{R} \mathscr{L})^{-1}}{0} & 0 \\
(I-\mathscr{L} \mathscr{R})^{-1}
\end{array}\right),
$$

and the estimate $(4.7 \mathrm{~b})$ follows:

$$
\left|E^{-1}(s)\right| \leqq\left(2+|\mathscr{R}|^{2}+|\mathscr{L}|^{2}\right)^{1 / 2} \cdot \frac{1}{\delta} \leqq \frac{1+|R|+|L|}{\delta} .
$$

Thus, the matrix $D$ in (4.6) is regular, being the product of regular matrices, and the lemma is proved.

The estimate $(4.10 \mathrm{~b})$ is, in fact, the last ingredient needed for a stability proof for (1.5). We can state

THEOREM 4.2 (Stability). Consider the hyperbolic system (1.1a)-(1.1e), satisfying the dissipativity Assumption I. Then its (pseudo)spectral approximation (1.5a)-(1.5c) is stable, provided that Assumptions II and III are fulfilled.

Proof. System (1.5a) consists of $l$ inflow equations

$$
s \hat{v}^{(j)}=a_{j} \frac{d \hat{v}^{(j)}}{d x}+q^{I}(x) \tau^{(j)}, \quad 1 \leqq j \leqq l
$$


and $n-l$ outflow ones

$$
s \hat{v}^{(j)}=a_{j} \frac{d \hat{v}^{(j)}}{d x}+q^{I I}(x) \tau^{(j)}, \quad l<j \leqq n .
$$

By the scalar stability Assumption II, each of these scalar equations is stable. That is, there exist a weighting pair $\omega(x)=\left(\omega^{I}(x), \omega^{I I}(x)\right)^{\prime}$ and constants $\alpha$ and $\eta_{0} \geqq 0$ such that for all $s$ with $\operatorname{Re} s=\eta>\eta_{0}$ we have for arbitrary vectors $\tau$

$$
\begin{array}{ll}
\left(\eta-\eta_{0}\right)\left\|v_{N}^{(j)}(x, s)\right\|_{\omega}^{2} \leqq \text { const } N^{2 \alpha}\left|\hat{v}_{N}^{(j)}(-1, s)\right|^{2}, & 1 \leqq j \leqq l, \\
\left(\eta-\eta_{0}\right)\left\|v_{N}^{(j)}(x, s)\right\|_{\omega}^{2}{ }^{2 I} \text { const } N^{2 \alpha}\left|\hat{v}_{N}^{(j)}(1, s)\right|^{2}, & l<j \leqq n .
\end{array}
$$

Using the spatial norm of (1.2a),

$$
\left\|\hat{\mathbf{v}}_{N}(x, s)\right\|^{2} \equiv\left\|\hat{\mathbf{v}}_{N}(x, s)\right\|_{\omega}^{2}=\left\|\hat{v}_{N}^{I}(x, s)\right\|_{\omega^{I}}^{2}+\left\|\hat{v}_{N}^{I I}(x, s)\right\|_{\omega^{I I}}^{2}
$$

these inequalities can be added together and rewritten in concise form

$$
\left(\eta-\eta_{0}\right)\left\|\hat{\mathbf{v}}_{N}(x, s)\right\|^{2} \leqq \text { const } N^{2 \alpha}\left[\left|\hat{\mathbf{v}}_{N}^{I}(-1, s)\right|^{2}+\left|\hat{\mathbf{v}}_{N}^{I I}(1, s)\right|^{2}\right], \quad \operatorname{Re} s=\eta>\eta_{0} \geqq 0 .
$$

It remains to estimate the two squared boundary terms inside the brackets on the right. To this end we rewrite our solution in the form (cf. (4.5) and (4.11))

$$
\hat{\mathrm{v}}_{N}^{I}(x, s)=P^{I}(x, s) \tau^{I}, \quad \hat{\mathrm{v}}_{N}^{I I}(x, s)=P^{I I}(x, s) \tau^{I I} .
$$

Inserting this in the boundary conditions $(1.5 \mathrm{c})$, we find, as in (3.5), that $\tau=\tau(s)$ is determined uniquely by

$$
\left[\begin{array}{c}
\tau^{I} \\
\tau^{I I}
\end{array}\right]=D^{-1}(s)\left[\begin{array}{c}
\hat{\mathbf{g}}^{I}(s) \\
\hat{\mathbf{g}}^{I I}(s)
\end{array}\right]
$$

Using (4.14), the quantities we want to estimate can be expressed in terms of the vector $\tau$

$$
\left[\begin{array}{c}
\hat{\mathrm{v}}_{N}^{I}(-1, s) \\
\hat{\mathrm{v}}_{N}^{I I}(1, s)
\end{array}\right]=\left[\begin{array}{cc}
P^{I}(-1, s) & 0 \\
0 & P^{I I}(1, s)
\end{array}\right]\left[\begin{array}{c}
\tau^{I} \\
\tau^{I I}
\end{array}\right]
$$

From formulas (4.6) and (4.16) we deduce

$$
\left[\begin{array}{c}
\hat{\mathbf{v}}_{N}^{I}(-1, s) \\
\hat{\mathbf{v}}_{N}^{I I}(1, s)
\end{array}\right]=E^{-1}(s)\left[\begin{array}{c}
\hat{\mathbf{g}}^{I}(s) \\
\hat{\mathbf{g}}^{I I}(s)
\end{array}\right]
$$

and, since by (4.7b) $E(s)$ has a uniformly bounded inverse, we end up with

$$
\left|\hat{\mathbf{v}}_{N}^{I}(-1, s)\right|^{2}+\left|\hat{\mathbf{v}}_{N}^{I I}(1, s)\right|^{2} \leqq K|\mathbf{g}(s)|^{2}, \quad \operatorname{Re} s=\eta>\eta_{0} \geqq 0 .
$$

Thus, (4.13) and (4.18) add up to (1.14), which is the definition of stability.

We note that once factorized as in (4.6), the boundary matrix $D(s)$ is invertible, if and only if the matrix $E(s)$ is. Both Assumptions I and III were introduced in order to further guarantee that $E(s)$ is uniformly bounded; in fact, one could assume, instead, that an $s$-uniform estimate like (4.7b) holds. This corresponds to the Uniform Kreiss Condition which characterizes the stability of difference approximations to initialboundary value systems. The merit of Assumption III, however, is that it deals with the scalar problem only, rather than with the intricate coupled boundary matrix $E(s)$.

5. The Chebyshev, Legendre, and Gegenbauer pseudospectral methods. In this section we address ourselves to the question of the stability of the most commonly used pseudospectral methods, namely Legendre and Chebyshev collocation. We employ the 
stability criterion stated in Theorem 4.2 , i.e., we shall verify that the scalar stability Assumptions II and III hold.

The scalar model is, of course,

$$
s \hat{v}_{N}=a \frac{d \hat{v}_{N}}{d x}+q(x) \tau(s)
$$

with $\hat{\mathrm{v}}$ given at $x=1(-1)$ if $a>0(a<0)$, respectively.

Our results may be stated in a more general context, involving Gegenbauer polynomials, of which Chebyshev and Legendre polynomials are particular cases. The Gegenbauer polynomials $C_{N}^{\alpha+(1 / 2)}$ are (suitably normalized) orthogonal polynomials with respect to the weight

$$
\omega(x)=\left(1-x^{2}\right)^{\alpha}
$$

in the interval $-1 \leqq x \leqq 1$. Thus, the Legendre polynomial $P_{N}$ is a multiple of $C_{N}^{1 / 2}$, and the Chebyshev polynomial $T_{N}$ is a multiple of $C_{N}^{0}$.

We shall make extensive use of the Lobatto quadrature rule, which is valid whenever $p(x)$ is a polynomial of degree $<2 N+2$ :

$$
\int_{-1}^{1} \omega(x) p(x) d x=\sum_{j=0}^{N+1} \omega_{j} p\left(x_{j}\right), \quad \omega_{j}>0 .
$$

Here, $x_{0}=1$ and $x_{N+1}=-1$ denote the endpoints of the interval of integration and the interior points

$$
1>x_{1}>x_{2}>\cdots x_{N-1}>x_{N}>-1
$$

are the zeros of the Gegenbauer polynomial $C_{N}^{\alpha+(3 / 2)}$. In particular, for the Chebyshev method these points are the zeros of $T_{N+1}^{\prime}$, while for the Legendre method they are the zeros of $P_{N+1}^{\prime}$-this follows from the identity

$$
\frac{d}{d x} C_{N}^{\alpha}=2 \alpha C_{N-1}^{\alpha+1}
$$

We consider collocating the equations at the interior points $x_{j}, 1 \leqq j \leqq N$. Then the forcing polynomial $q(x)$ is proportional to $C_{N}^{\alpha+(3 / 2)}$ and may be normalized as

$$
q(x)=\frac{C_{N}^{\alpha+(3 / 2)}(x)}{C_{N}^{\alpha+(3 / 2)}(1)}
$$

in order to have $q(1)=1$.

THEOREM 5.1 (Scalar stability). Let $-1<\alpha \leqq 0$; then we have for $s$ with $0<\operatorname{Re} s=$ $\eta<\boldsymbol{H}$

$$
\begin{array}{ll}
\eta \int_{-1}^{1}(1+x) \omega(x)|\hat{v}(x, s)|^{2} d x \leqq \text { const } A(N)|\hat{v}(1, s)|^{2}, & a>0, \\
\eta \int_{-1}^{1}(1-x) \omega(x)|\hat{v}(x, s)|^{2} d x \leqq \text { const } A(N)|\hat{v}(-1, s)|^{2}, & a<0 .
\end{array}
$$

Here, $A(N)$ is a power growth bound:

$$
A(N)= \begin{cases}N^{-2 \alpha}, & -1<\alpha<0, \\ N^{2}, & \alpha=0 \quad \text { (Legendre), }\end{cases}
$$


and $H=H(\alpha)$ may be taken independent of $N$. For this collocation method, the weighting pair, as required in formu $\mathrm{a}$ (1.2a), is

$$
\omega^{I}=(1-x)\left(1-x^{2}\right)^{\alpha}, \quad \omega^{I I}=(1+x)\left(1-x^{2}\right)^{\alpha} .
$$

Proof. Let us study first the case $a>0$-without loss of generality we may take $a=1$. Consider the $N$ th degree polynomial

$$
z(x, s)=\hat{v}(x, s)-q(x) \hat{v}(1, s) .
$$

We have

$$
\begin{gathered}
z\left(x_{j}, s\right)=\hat{v}\left(x_{j}, s\right), \quad 1 \leqq j \leqq N, \\
z(1, s)=0
\end{gathered}
$$

and, in view of (5.1):

$$
s z\left(x_{j}, s\right)=\frac{d}{d x} z\left(x_{j}, s\right)+\hat{v}(1, s) q^{\prime}\left(x_{j}\right) \quad \text { for } 1 \leqq j \leqq N .
$$

Multiply (5.9c) by $\left(1+x_{j}\right) z^{*}\left(x_{j}, s\right) \omega_{j}$ and sum the real parts (terms with $j=0$ and $j=N+1$ can be included, as their contribution vanishes)

$$
\begin{aligned}
\sum_{j=1}^{N} \eta \omega_{j}\left(1+x_{j}\right)\left|z\left(x_{j}, s\right)\right|^{2}= & \sum_{j=0}^{N+1} \frac{1}{2} \omega_{j}\left(1+x_{j}\right)\left(\frac{d}{d x}|z|^{2}\right)\left(x_{j}, s\right) \\
& +\operatorname{Re}\left(\hat{v}(1, s) \cdot \sum_{j=0}^{N+1} \omega_{j}\left(1+x_{j}\right) z^{*}\left(x_{j}, s\right) q_{N}^{\prime}\left(x_{j}\right)\right)
\end{aligned}
$$

On the left of this equality we have $z\left(x_{j}, s\right)=v\left(x_{j}, s\right)$, while the sums on the right are in fact integrals, according to formula (5.3). Thus, we obtain

$$
\begin{aligned}
\eta \sum_{j=1}^{N}\left(1+x_{j}\right) \omega_{j}\left|v\left(x_{j}, s\right)\right|^{2}= & \int_{-1}^{1} \frac{1}{2}(1+x) \omega \frac{d}{d x}|z|^{2} d x \\
& +\operatorname{Re}\left(\hat{v}(1, s) \cdot \int_{-1}^{1}(1+x) \omega z^{*} q_{N}^{\prime} d x\right) .
\end{aligned}
$$

Integrating by parts the first term on the right-hand side and using the Cauchy-Schwarz inequality on the second, we conclude that

$$
\begin{aligned}
\eta \sum_{j=1}^{N}(1 & \left.+x_{j}\right) \omega_{j}\left|v\left(x_{j}, s\right)\right|^{2} \\
= & \left.\frac{1}{2}(1+x) \omega|z|^{2}\right|_{-1} ^{1}-\int_{-1}^{1} \frac{1}{2}((1+x) \omega)^{\prime}|z|^{2} d x \\
& +\operatorname{Re}\left(\hat{v}(1, s) \cdot \int_{-1}^{1}(1+x) \omega z^{*} q_{N}^{\prime} d x\right) \\
\leqq & -\int_{-1}^{1} \frac{1}{2}((1+x) \omega)^{\prime}|z|^{2}+\int_{-1}^{1} \frac{1}{2}((1+x) \omega)^{\prime}|z|^{2} \\
& +2 \cdot|\hat{v}(1, s)|^{2} \cdot \int_{-1}^{1} \frac{\left((1+x) \omega q_{N}^{\prime}\right)^{2}}{((1+x) \omega)^{\prime}} d x \\
= & 2 \cdot|\hat{v}(1, s)|^{2} A(N)
\end{aligned}
$$


where

$$
A(N)=\int_{-1}^{1} \frac{\left((1+x) \omega(x) q_{N}^{\prime}(x)\right)^{2}}{((1+x) \omega(x))^{\prime}} d x
$$

Note that by adding the boundary terms to the sum on the left, it can be replaced by the corresponding integral in (5.7) and the inequality (5.12) still holds for $|\eta| \leqslant A(N)$. Also notice that all the integrals involved are proper integrals, because of the limitations on $\alpha$. It remains to show that $A(N)$ is bounded by some power of $N$. A coarse bound can be obtained immediately, by remarking that

$$
\max _{|x| \leqq 1}\left|C_{N}^{\lambda}(x)\right|=C_{N}^{\lambda}(1) \text { for } \lambda>0,
$$

and therefore $|q(x)|$ is bounded by 1 in $-1 \leqq x \leqq 1$. By a well-known extremal property of Chebyshev polynomials [6, Thm. 2.24], the derivatives cannot grow faster than the corresponding derivatives of $T_{N}$, and, thus, $\left|q^{\prime}\right| \leqq N^{2}$. Hence,

$$
A(N) \leqq N^{4} F(\alpha), \quad F(\alpha)=\int_{-1}^{1} \frac{(1+x)^{\alpha+2}(1-x)^{\alpha+1}}{1-(2 \alpha+1) x} d x
$$

The more delicate estimates of (5.7c) are relegated to Appendix B. The proof of (5.7b) follows along similar lines.

We now show that the collocation methods we have described satisfy Assumption III.

THEOREM 5.2. Let $\hat{v}$ be a polynomial in $x$ which satisfies

$$
s \hat{v}(x, s)=\frac{d}{d x} \hat{v}(x, s)+q(x)
$$

where $q(x)$ is defined by (5.6). If $\hat{v}\left(x=1, s_{0}\right)=0$, then $\operatorname{Re} s_{0}<0$. In fact, $\hat{v}$ satisfies (4.1b), i.e., Assumption III holds.

Proof. In a manner similar to (5.10)-(5.12) we can deduce

$$
\begin{gathered}
\operatorname{Re} s_{0}\left(1+x_{j}\right) \omega_{j}\left|\hat{v}\left(x_{j}, s_{0}\right)\right|^{2}=\left(1+x_{j}\right) \omega_{j} \frac{d}{d x}|\hat{v}|^{2}\left(x_{j}, s_{0}\right), \quad 1 \leqq j \leqq N, \\
\operatorname{Re} s_{0} \int_{-1}^{1}(1+x) \omega|\hat{v}|^{2} d x=\int_{-1}^{1}(1+x) \omega \frac{d}{d x}|\hat{v}|^{2} d x=-\int_{-1}^{1}|v|^{2}((1+x) \omega)^{\prime} d x .
\end{gathered}
$$

From the last formula, it is obvious that $\operatorname{Re} s_{0}<0$. Because of the symmetry of $q(x)$,

$$
q(x) \equiv q_{N}(x)=(-1)^{N} q_{N}(-x),
$$

it is clear that $\hat{v}(-1, s)=(-1)^{N} \hat{v}(1,-s)$. Consider now the rational function of $s$, $\operatorname{Re} s \geqq 0$, with real coefficients

$$
f(s)=\frac{\hat{v}(1,-s)}{\hat{v}(1 ; s)}
$$

The limit of $|f(s)|$ exists uniformly as $s \rightarrow \infty$ and equals one, while on the imaginary axis

$$
|f(s)|=1 \text {, }
$$

being the quotient of a number and its conjugate. Therefore, since

$$
f(s)=(-1)^{N} \frac{\hat{v}(-1, s)}{\hat{v}(1, s)}
$$

is regular for $\operatorname{Re} s \geqq 0$, it is bounded by one in magnitude-which is Assumption III. 
With minor modifications the same proof covers the case $a<0$.

Finally, we can summarize this section by applying Theorem 4.2 to the scalar results we have obtained thus far.

THEOREM 5.3 (Stability of Gegenbauer Collocation for Systems). Consider the hyperbolic system (1.1), satisfying Assumption I. Discretize it by collocating at the zeros of $C_{N}^{\alpha+(3 / 2)}$, where $-1<\alpha \leqq 0$. The resulting spectral method is stable, in the sense of Lemma 1.2, with parameters defined by (5.7c) and (5.7d). In particular, Chebyshev and Legendre collocation methods are stable.

Appendix A. In this section we prove several a priori bounds for the solution of the differential system (1.1) and its scalar counterparts.

Introducing into (1.1) the new variable $e^{-\eta t} u(x, t)$ and taking the Fourier transform with respect to time, we obtain the equations

$$
\begin{aligned}
s \hat{\mathbf{u}}^{I} & =A^{I} \frac{d \hat{\mathbf{u}}^{I}}{d x}, \\
s \hat{\mathbf{u}}^{I I} & =A^{I I} \frac{d \hat{\mathbf{u}}^{I I}}{d x},
\end{aligned}
$$

with boundary conditions

$$
\begin{aligned}
& \hat{\mathbf{u}}^{I}(-1, s)=L \hat{\mathbf{u}}^{I I}(-1, s)+\hat{\mathbf{g}}^{I}(s), \\
& \hat{\mathbf{u}}^{I I}(1, s)=R \hat{\mathbf{u}}^{I}(1, s)+\hat{\mathbf{g}}^{I I}(s) .
\end{aligned}
$$

Set $\omega^{I}(x)=1+\varepsilon x$ with $0 \leqq \varepsilon \leqq 1$ yet to be determined, and multiply (A.1a) by $\omega^{I} \hat{\mathbf{u}}^{I^{*}}$. After integration by parts, we find

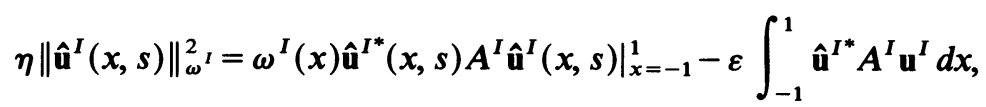

where $\eta=\operatorname{Re} s$. Since $\varepsilon A^{I}$ is negative, the second term on the right-hand side does not exceed $(\varepsilon /(1-\varepsilon))|A|\left\|\hat{\mathbf{u}}^{I}(x, s)\right\|_{\omega^{I}}^{2}$ and hence

$$
\left(\eta-\eta_{0}\right)\left\|\hat{\mathbf{u}}^{I}(x, s)\right\|_{\omega^{I}}^{2} \leqq\left.(1+\varepsilon x) \hat{\mathbf{u}}^{I^{*}}(x, s) A^{I} \hat{\mathbf{u}}^{I}(x, s)\right|_{x=-1} ^{1}
$$

with $\eta_{0}=(\varepsilon /(1-\varepsilon))|A|$.

We can draw two conclusions from (A.3). First, by applying it to each of the scalar inflow equations separately, we have for all $s$ with $\operatorname{Re} s=\eta>\eta_{0}$, a positive left-hand side

$$
0<a_{j}\left[(1+\varepsilon)\left|\hat{u}^{(j)}(1, s)\right|^{2}-(1-\varepsilon)\left|\hat{u}^{(j)}(-1, s)\right|^{2}\right], \quad 1 \leqq j \leqq l,
$$

that is, as $a_{j}<0$

$$
\left|\frac{U^{I}(1, s)}{U^{I}(-1, s)}\right| \leqq \frac{1-\varepsilon}{1+\varepsilon}, \quad U^{I}=\left[\begin{array}{lll}
\hat{u}^{(1)}(x, s) & & \\
& \ddots & \\
& & \hat{u}^{(l)}(x, s)
\end{array}\right] .
$$

Second, since $(1+\varepsilon x) A^{I}$ is negative, the inequality (A.3) yields

$$
\left(\eta-\eta_{0}\right)\left\|\hat{\mathbf{u}}^{I}(x, s)\right\|_{\omega^{I}}^{2}(1-\varepsilon)\left|A^{I}\right|\left|\hat{\mathbf{u}}^{I}(-1, s)\right|^{2} .
$$

We can treat similarly the outflow part of the system. Choosing $\omega^{I I}=1-\varepsilon x$, we find that

$$
\left|\frac{U^{I I}(-1, s)}{U^{I I}(1, s)}\right| \leqq \frac{1-\varepsilon}{1+\varepsilon}, \quad U^{I I}=\left[\begin{array}{lll}
\hat{u}^{(l+1)}(x, s) & & \\
& \ddots & \\
& & \hat{u}^{(n)}(x, s)
\end{array}\right],
$$


and

$$
\left(\eta-\eta_{0}\right)\left\|\hat{\mathbf{u}}^{I I}(x, s)\right\|_{\omega^{I I}}^{2} \leqq(1-\varepsilon)\left|A^{I I}\right|\left|\hat{\mathbf{u}}^{I I}(1, s)\right|^{2} .
$$

Finally, we rewrite the boundary conditions in the form

$$
\left(\begin{array}{cc}
I & -L \frac{U^{I I}(-1, s)}{U^{I I}(1, s)} \\
-R \frac{U^{I}(1, s)}{U^{I}(-1, s)} & I
\end{array}\right)\left(\begin{array}{c}
\hat{\mathbf{u}}^{I}(-1, s) \\
\hat{\mathbf{u}}^{I I}(1, s)
\end{array}\right)=\left(\begin{array}{c}
\hat{\mathbf{g}}^{I}(s) \\
\hat{\mathbf{g}}^{I I}(s)
\end{array}\right), \quad \operatorname{Re} s=\eta>\eta_{0} .
$$

As in Lemma 4.1, the matrix on the left is invertible; in view of (A.4) and (A.6) the inverse is bounded in the norm

$$
\left|\left(\begin{array}{cc}
I & -L \frac{U^{I I}(-1, s)}{U^{I I}(1, s)} \\
-R \frac{U^{I}(1, s)}{U^{I}(-1, s)} & I
\end{array}\right)^{-1}\right| \leqq \frac{1+(|R|+|L|) \frac{1-\varepsilon}{1+\varepsilon}}{1-|R||L|\left(\frac{1-\varepsilon}{1+\varepsilon}\right)^{2}},
$$

provided $\varepsilon$ is chosen so that the denominator is positive. Then we have

$$
\left|\hat{\mathbf{u}}^{I}(-1, s)\right|^{2}+\left|\hat{\mathbf{u}}^{I I}(1, s)\right|^{2} \leqq \text { const }\left[\left|\hat{\mathbf{g}}^{I}(s)\right|^{2}+\left|\hat{\mathbf{g}}^{I I}(s)\right|^{2}\right],
$$

and together with (A.5) and (A.7) we reach

$$
\left(\eta-\eta_{0}\right)\|\hat{\mathbf{u}}(x, s)\|^{2} \leqq \text { const }|\hat{\mathbf{g}}(s)|^{2}, \quad \operatorname{Re} s=\eta>\eta_{0} .
$$

Hence, the energy estimate referred to in (1.2b) follows from Parseval's relation, with any $\eta_{0}$ satisfying

$$
\eta_{0}=\frac{\varepsilon}{1-\varepsilon}|A| \geqq \frac{(|R||L|)^{1 / 2}-1}{2(|R||L|)^{1 / 2}}|A| .
$$

We conclude by noting that in the dissipative case, where $|R||L|<1$, one may take $\eta_{0}=\varepsilon=0$, and the formulas (A.4), (A.6) then lead to

$$
\begin{array}{ll}
\left|\hat{u}^{(j)}(1, s)\right| \leqq\left|\hat{u}^{(j)}(-1, s)\right|, & 1 \leqq j \leqq l, \\
\left|\hat{u}^{(j)}(-1, s)\right| \leqq\left|\hat{u}^{(j)}(1, s)\right|, & l<j \leqq n,
\end{array}
$$

for $\operatorname{Re} s=\eta>0$.

Appendix B. Here we evaluate (5.13), in order to obtain the asymptotic behavior of our estimates for large $N$. We shall compute

$$
A(N)=\int_{-1}^{1} \frac{\left((1+x) \omega q_{N}^{\prime}\right)^{2}}{((1+x) \omega)^{\prime}} d x=\int_{-1}^{1} \frac{\left[(1+x)\left(1-x^{2}\right)^{\alpha}\left(C_{N+1}^{\alpha+(1 / 2)}(x)\right)^{\prime \prime}\right]^{2}}{\left[(1+x)\left(1-x^{2}\right)^{\alpha}\right]^{\prime}\left[\left(C_{N+1}^{\alpha+(1 / 2)}\right)^{\prime}(1)\right]^{2}} d x
$$

separately for $\alpha=0$, the Legendre spectral method, and for $-1<\alpha<0$. In the first case, we integrate-by-parts

$$
\begin{aligned}
\int_{-1}^{1}(1+x)^{2}\left(P_{N+1}^{\prime \prime}\right)^{2} d x= & \left.P_{N+1}^{\prime}(x) P_{N+1}^{\prime \prime}(x)(1+x)^{2}\right|_{-1} ^{1} \\
& -\int_{-1}^{1} P_{N+1}^{\prime}\left(P_{N+1}^{\prime \prime}(1+x)^{2}\right)^{\prime} d x
\end{aligned}
$$


Once we obtain an integral involving $P_{N+1}^{\prime}$, we can use Lobatto quadrature to exploit the fact that $P_{N+1}^{\prime}$ vanishes at the interior nodes. Then use the explicit estimates

$$
\left(\frac{d^{m}}{d x^{m}} P_{N}\right)(1)=O\left(N^{2 m}\right)
$$

and

$$
\omega_{0}=O\left(N^{-2}\right)
$$

to deduce that $A(N)=O\left(N^{2}\right)$.

In the second case we proceed by first estimating

$$
\begin{aligned}
A(N) & =\int_{-1}^{1} \frac{(1+x)^{\alpha+2}(1-x)^{\alpha+1}}{1-(2 \alpha+1) x}\left[\frac{\left(C_{N+1}^{\alpha+(1 / 2)}\right)^{\prime \prime}(x)}{\left(C_{N+1}^{\alpha+(1 / 2)}\right)^{\prime}(1)}\right]^{2} d x \\
& \leqq \frac{1 / 2}{\min (|\alpha|, 1-|\alpha|)} \int_{-1}^{1}(1+x)^{\alpha+2}(1-x)^{\alpha}\left[\frac{\left(C_{N+1}^{\alpha+(1 / 2)}\right)^{\prime \prime}(x)}{\left(C_{N+1}^{\alpha+(1 / 2)}\right)^{\prime}(1)}\right]^{2} d x
\end{aligned}
$$

We then substitute from the Gegenbauer differential equation

$$
\left(1-x^{2}\right)\left(C_{N+1}^{\alpha+(1 / 2)}\right)^{\prime \prime}=2(\alpha+1) x\left(C_{N+1}^{\alpha+(1 / 2)}\right)^{\prime}-(N+1)(N+2 \alpha+2) C_{N+1}^{\alpha+(1 / 2)}
$$

to reach

$$
\begin{array}{r}
A(N) \sim \frac{1}{\left[\left(C_{N+1}^{\alpha+(1 / 2)}\right)^{\prime}(1)\right]^{2}}\left[\int_{-1}^{1}\left(1-x^{2}\right)^{\alpha}(1+x) 2(\alpha+1) x\left(C_{N+1}^{\alpha+(1 / 2)}\right)^{\prime}\left(C_{N+1}^{\alpha+(1 / 2)}\right)^{\prime \prime} d x\right. \\
-(N+1)(N+2 \alpha+2) \\
\left.\quad \cdot \int_{-1}^{1}\left(1-x^{2}\right)^{\alpha}(1+x) C_{N+1}^{\alpha+(1 / 2)}\left(C_{N+1}^{\alpha+(1 / 2)}\right)^{\prime \prime} d x\right] .
\end{array}
$$

In this expression, the second integral vanishes by orthogonality, and the first may be evaluated by Lobatto quadrature:

$$
A(N) \sim \frac{1}{\left[\left(C_{N+1}^{\alpha+1 / 2)}\right)^{\prime}(1)\right]^{2}} \cdot 2 \omega_{0} \cdot\left(C_{N+1}^{\alpha+(1 / 2)}\right)^{\prime \prime}(1)\left(C_{N+1}^{\alpha+(1 / 2)}\right)^{\prime}(1) .
$$

As $(1+x) C_{N}^{\alpha+(3 / 2)}$ vanishes at the nodes $x_{j}, 1 \leqq j<N+1$, we can compute $\omega_{0}$ :

$$
\omega_{0}=\int_{-1}^{1} \frac{\left(1-x^{2}\right)^{\alpha}(1+x) C_{N}^{\alpha+(3 / 2)}(x) d x}{2 C_{N}^{\alpha+(3 / 2)}(1)},
$$

which we estimate by the means of (5.14). Finally, we obtain

$$
A(N) \leqq O\left(N^{-2 \alpha}\right)
$$

in accordance with formula $(5.7 \mathrm{c})$.

\section{REFERENCES}

[1] P. J. DAVIS AND P. Rabinowitz, Methods of Numerical Integration, Academic Press, New York, 1975.

[2]. D. Gottlieb AND S. A. Orszag, Numerical Analysis of Spectral Methods: Theory and Applications, Society for Industrial and Applied Mathematics, Philadelphia, PA, 1977.

[3] R. G. Voigt, D. Gotrlieb AND M. Y. Hussaini, EDS., Spectral Methods for Partial Differential Equations, Society for Industrial and Applied Mathematics, Philadelphia, 1984.

[4] H. O. KREISS, Initial boundary value problems for hyperbolic systems, Comm. Pure Appl. Math., 23 (1970), pp. 277-298. 
[5] L. LuSTMAN, The time evolution of spectral discretizations of hyperbolic systems, this Journal, 23 (1986), pp. 1193-1198.

[6] T. J. RIVLIN, The Chebyshev Polynomials, Wiley-Interscience, New York, 1974.

[7] D. GotTLIEB AND E. TURKEL, Spectral methods for time-dependent partial differential equations, NASA Contractor Report 172241, October 1983.

[8] L. G. M. ReYNA, Ph.D. thesis, Part I, California Institute of Technology, Pasadena, CA, 1982. 